\title{
Depression and Anxiety during Pregnancy: The Influence of Maternal Characteristics
}

\author{
Kim FE van de Loo ${ }^{1 \# *}$, Richelle Vlenterie ${ }^{2 \#}$, Sylke J Nikkels ${ }^{3}$, Peter JFM Merkus ${ }^{4}$, Jolt \\ Roukema ${ }^{4}$, Chris M Verhaak ${ }^{1}$, Nel Roeleveld ${ }^{2,3}$ and Marleen MHJ van Gelder ${ }^{2,5}$
}

\author{
${ }^{1}$ Department of Medical Psychology, Radboudumc Amalia Children's Hospital, Radboud University Medical \\ Center, Nijmegen, The Netherlands
}

${ }^{2}$ Department for Health Evidence, Radboud Institute for Health Sciences, Radboud University Medical Center, Nijmegen, The Netherlands

${ }^{3}$ Department of Paediatrics, Radboudumc Amalia Children's Hospital, Radboud University Medical Center, Nijmegen, The Netherlands

${ }^{4}$ Department of Paediatric Pulmonology, Radboudumc Amalia Children's Hospital, Radboud University Medical Center, Nijmegen, The Netherlands

${ }^{5}$ Radboud REshape Innovation Center, Radboud University Medical Center, Nijmegen, The Netherlands

"Both authors contributed equally

\begin{abstract}
Introduction: Depression and anxiety during pregnancy are associated with adverse health outcomes for both mother and child. This study aimed to investigate the occurrence of symptoms of depression and anxiety in early and late pregnancy, the longitudinal changes from early to late pregnancy, and factors associated with symptoms of depression and anxiety in pregnant women in the Netherlands.

Methods: We studied 2897 women participating in the PRegnancy and Infant DEvelopment (PRIDE) Study. To assess symptoms of depression and anxiety, web-based questionnaires including the Hospital Anxiety and Depression Scale (HADS) and multiple questions on maternal characteristics, including demographics, health, and lifestyle factors, were completed in early and late pregnancy. Cross-sectional and longitudinal multivariable linear regression analyses were conducted.

Results: According to the HADS, the prevalence of depression in our population increased from $5.4 \%$ in early pregnancy to $10.0 \%$ in late pregnancy $(\mathrm{p}<0.001$ ), whereas the prevalence of anxiety decreased from $17.9 \%$ to $14.2 \%(p<0.001)$. Several maternal characteristics, such as level of education, multiparity, a history of depression, severe nausea, extreme fatigue, lack of physical exercise, and negative life events were associated with symptoms of depression and/or anxiety throughout pregnancy. Being non-Dutch, not living with a partner, and having an unplanned pregnancy or a prolonged time to pregnancy influenced the depression and/or anxiety scores in early pregnancy only.

Conclusions: Depression and anxiety are common disorders in both early and late pregnancy. Screening for risk factors for these disorders in early pregnancy is important, since prenatal depression and anxiety may be related to adverse maternal and child health outcomes.

Keywords

Depression, Anxiety, Pregnancy, Maternal exposure, PRIDE Study, Psychological epidemiology
\end{abstract}

\section{Introduction}

Maternal depression and anxiety during pregnancy are an important research topic, as both have been associated with a broad range of adverse health outcomes for mother and child [1-8]. Several studies found associations between maternal depression and/or anxiety and either lower birth weight or shorter gestational age or both [4,9-12]. Since these outcomes may lead to increased childhood mortality and long-term morbidity, $[13,14]$ they may have an enormous impact on public health and future health care. Furthermore, maternal depression and anxiety may have negative

Copyright: ( 2017 van de Loo KFE, et al. This is an open-access article distributed under the terms of the Creative Commons Attribution License, which permits unrestricted use, distribution, and reproduction in any medium, provided the original author and source are credited. 
Citation: van de Loo KFE, Vlenterie R, Nikkels SJ, et al. (2017) Depression and Anxiety during Pregnancy: The Influence of Maternal Characteristics. J Mood Disord Ther 1(1):1-16

consequences for, amongst others, social, behavioral, and neurodevelopment of the child [6,15-17]. Furthermore, prenatal depression and anxiety have also been associated with adverse health outcomes for the mother, such as preeclampsia, decreased quality of life, and have been linked to an increased risk of postpartum depression [18-26]. Women with high levels of depressive symptoms in late pregnancy may not only show more symptoms of depression directly after birth, but even after 14 years of follow-up [27-28].

Data regarding prevalence rates of depression and anxiety and changes over time during pregnancy vary widely. In general, prevalence rates of anxiety seem to be higher compared to prevalence rates of depression [29-32]. For the latter, some reported a decrease from $11.0 \%$ to $8.5 \%$ over the course of pregnancy [33], while others reported an increase from $7.4 \%$ in early pregnancy to $12.8 \%$ in middle, and $12.0 \%$ in late pregnancy [34], or a U-shaped form with the lowest rate around 24 weeks of gestation [31]. Prevalence rates of anxiety and changes over time during pregnancy have been studied less extensively compared to depression, with prevalence rates ranging from $15.0 \%$ to $36.3 \%[29,31,32,35]$. Some reported an increase in anxiety [36], while others reported a U-shaped pattern [31,37], or a decrease over the course of pregnancy [38]. Differences in prevalence rates could be explained by heterogeneity among studies, for example due to differences in sociodemographic background [34]. Furthermore, depression and anxiety were assessed in different ways, for example, based on questionnaires or structured interviews, or on a diagnosis made by a professional.

As prenatal depression and anxiety seem to be strongly associated with adverse maternal and child-health outcomes, they are an important public health concern. Specific information regarding prevalence rates, longitudinal changes, and risk factors is necessary to provide adequate counseling and therapy during pregnancy to prevent adverse health outcomes for mother and child. Therefore, this exploratory study aimed to investigate the prevalence rates of symptoms of depression and anxiety in early and late pregnancy, as well as changes over time from early to late pregnancy in a large sample of pregnant women. Furthermore, we examined the associations of several maternal characteristics with symptoms of depression and anxiety during pregnancy.

\section{Materials and Methods}

\section{General design PRIDE study}

This study was embedded in the PRegnancy and Infant DEvelopment (PRIDE) Study, an ongoing prospective cohort study in the Netherlands. The PRIDE Study aims to include a large number of pregnant Dutch women in early pregnancy to answer a broad range of research questions pertaining to maternal and child health and development.
More details concerning the rationale and design of the PRIDE Study are reported elsewhere [39]. In short, pregnant women were invited to participate in the study by their prenatal care provider (i.e. midwife, gynecologist, or general practitioner) at the first prenatal care visit. After providing informed consent, participating women completed three web-based questionnaires during pregnancy (around gestational weeks 10-12,17, 34), as well as biannually throughout childhood. The questionnaires included questions on general maternal characteristics, chronic and pregnancy-related conditions, medication use, lifestyle, life events, and other exposures during pregnancy. Exclusion criteria are maternal age below 18 years, more than 16 weeks pregnant at intake, and inability to read and understand the Dutch language. The PRIDE Study was approved by the Regional Committee on Research involving Human Subjects Arnhem-Nijmegen. PRIDE Study participants with an estimated date of delivery from February 2012 through December 2015 were included in the present study. If a woman participated more than once in the PRIDE Study, only the first pregnancy was included.

\section{Measurement tool}

Hospital Anxiety Depression Scale (HADS): The PRIDE Study questionnaires in early (weeks 10-12) and late (week 34) pregnancy included the Hospital Anxiety Depression Scale (HADS), a 14-item self-completion screening scale to indicate the possible presence of symptoms of depression and anxiety [40]. The questions assess the experience of symptoms in the past week. The questionnaire consists of two subscales, one for symptoms of depression (HADS-D) and one for symptoms of anxiety (HADS-A), with a 4-point rating scale of severity of symptoms (0-3), adding up to a score range of $0-21$ for each subscale. The HADS is a commonly used questionnaire to screen for depression and anxiety both in out-patient clinics and in research settings and is validated in different groups of the Dutch population [41]. A cut-off score $\geq 8$ was used for both subscales to identify depression and anxiety $[40,42]$.

\section{Maternal characteristics}

Several maternal characteristics were included in this study to examine their associations with the HADS scores.

*Corresponding author: Kim FE van de Loo, Department of Medical Psychology, Radboudumc Amalia Children's Hospital, Radboud University Medical Center, Nijmegen, The Netherlands, Tel:+31-0-24-361-3947, Fax: +31-0-24-3613425, E-mail:Kim.vandeLoo@radboudumc.nl

Received: January 12, 2017; Accepted: April 12, 2017; Published online: April 15, 2017

Citation: van de Loo KFE, Vlenterie R, Nikkels SJ, et al. (2017) Depression and Anxiety during Pregnancy: The Influence of Maternal Characteristics. J Mood Disord Ther $1(1): 1-16$ 
Citation: van de Loo KFE, Vlenterie R, Nikkels SJ, et al. (2017) Depression and Anxiety during Pregnancy: The Influence of Maternal Characteristics. J Mood Disord Ther 1(1):1-16

Table 1: Maternal characteristics studied.

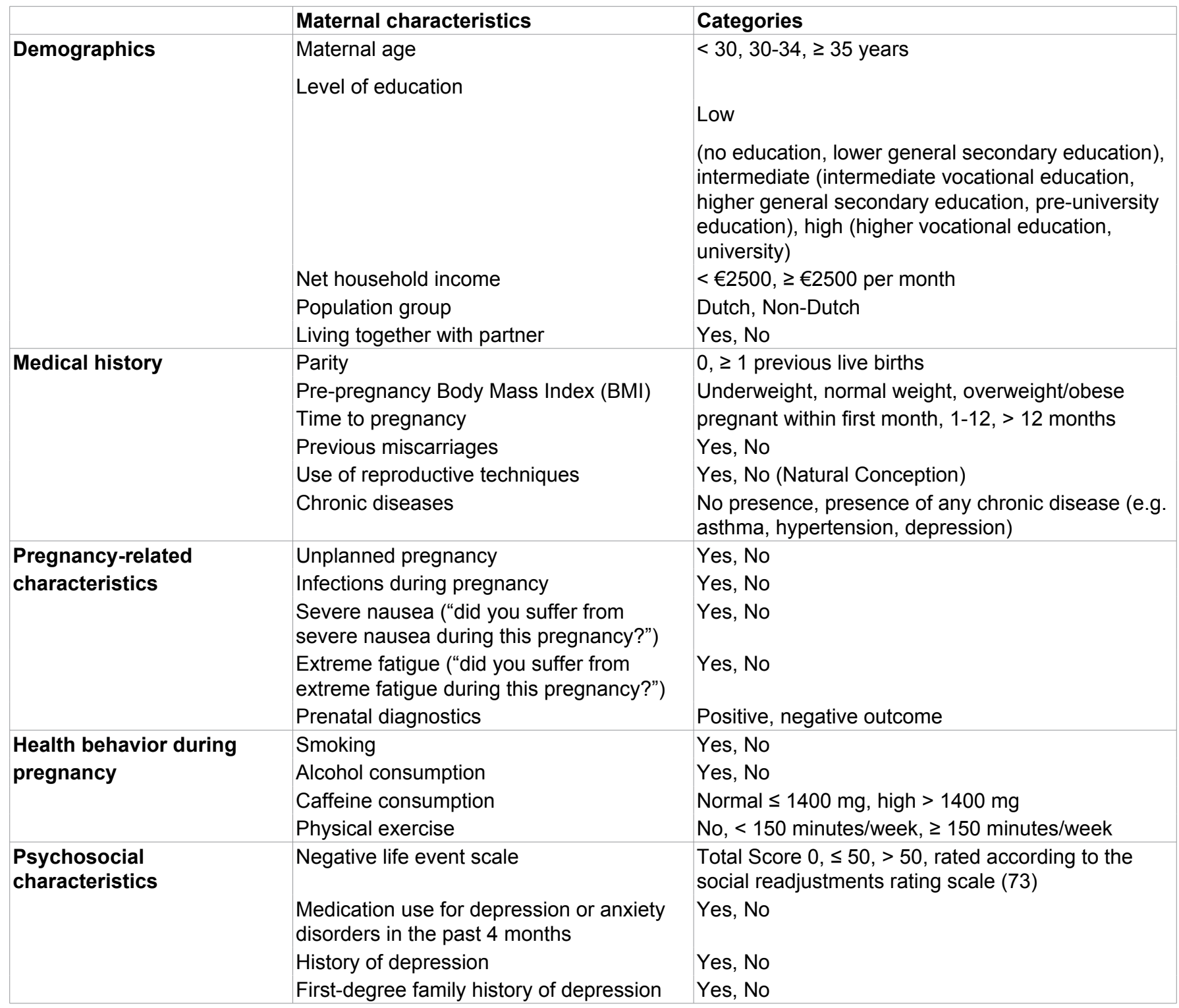

These characteristics were selected based on literature research and discussions among the authors, and were categorized into demographics, medical history, pregnancy-related characteristics, health behavior during pregnancy, and psychosocial characteristics. The specific characteristics within each category are listed in (Table 1). All maternal characteristics were self-reported and obtained from the three prenatal PRIDE Study questionnaires.

\section{Statistical analyses}

Based on the HADS-D and HADS-A scores, prevalence rates of depression and anxiety (scores $\geq 8$ ) in our population, as well as mean scores with Standard Deviations (SD) were calculated. Absolute numbers with percentages were used to describe the maternal characteristics, stratified by the presence of depression or anxiety according to the HADS-D and HADS-A, for both early and late pregnancy. To examine changes over time in the HADS-D and HADS-A scores from early to late pregnancy, multivariable linear mixed models with the subjects as random effects with manual backward elimination were applied, using p-values below 0.10 to retain all potentially relevant maternal characteristics in the final models.

Following, we fitted three linear regression models for each of the continuous outcome variables (HADS-D score and HADS-A score): two cross-sectional models, one in early pregnancy and one in late pregnancy, and one longitudinal model. For the cross-sectional models, univariable linear regression analyses were initially conducted for all selected maternal characteristics separately to obtain crude $\beta$ coefficients ( $\beta$ ) with $95 \%$ confidence intervals (95\% CI). Maternal characteristics that were stable over time, such as education and ethnicity, were included in both models, whereas characteristics measured in early pregnancy were included in the cross-sectional model for early pregnancy only, and characteristics measured in late pregnancy in the model for late pregnancy. Using complete case analyses, adjusted $\beta$ coefficients were derived from multivariable 
Citation: van de Loo KFE, Vlenterie R, Nikkels SJ, et al. (2017) Depression and Anxiety during Pregnancy: The Influence of Maternal Characteristics. J Mood Disord Ther 1(1):1-16

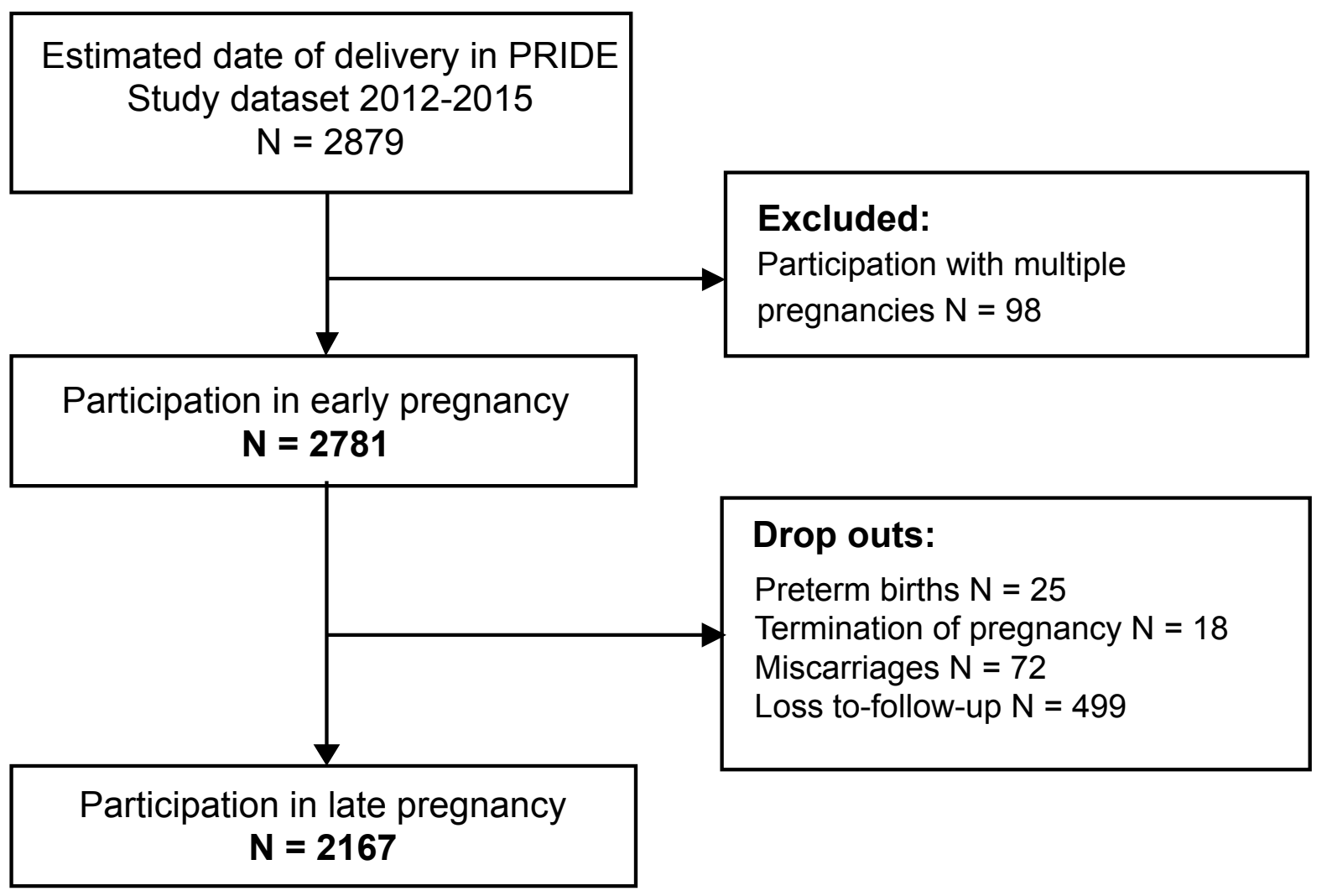

Figure 1: Flow chart displaying the women participating in the PRIDE Study and included in this study for analyses regarding early and late pregnancy.

linear regression models initially including all maternal characteristics. Manual stepwise backward elimination was applied to retain only variables with p-values below 0.10 in the models. Thereby, these characteristics were adjusted for all other maternal characteristics in the final models.

For the longitudinal models, multivariable linear regression analyses were done with the HADS-D and HADS-A scores measured in late pregnancy as outcome variables and all maternal characteristics measured in early and late pregnancy as potential determinants. Again, manual stepwise backward elimination was applied to identify all relevant risk factors for symptoms of depression and anxiety in late pregnancy. All statistical analyses were performed using the Statistical Package for Social Sciences, version 22.0 for Windows (IBM Corp., Armonk, NY).

\section{Results}

In total, 2897 women were included in this study, of which 2167 women completed the questionnaires in both early and late pregnancy (Figure 1). All results are based on the complete sample of women who filled out the HADS in the specific pregnancy period, except for the results of the longitudinal analyses.

\section{Prevalence rates}

Based on the HADS scores, the prevalence of de- pression in our population was $5.4 \%$ in early pregnancy (HADS-D mean score: 2.63, SD: 2.50) and 10.0\% in late pregnancy (HADS-D mean: 3.66, SD: 2.66). The mixed model analysis showed an increase in HADS-D scores throughout pregnancy (adjusted $\beta$ 1.50, 95\% CI: 1.31-1.68). The prevalence of anxiety was $17.9 \%$ in early pregnancy (HADS-A mean: 5.69, SD: 2.19) and 14.2\% in late pregnancy (HADS-A mean: 5.35, SD: 2.17), which was consistent with a decrease throughout pregnancy in HADS-A scores in the mixed model (adjusted $\beta-0.21$, 95\% CI: -0.38- - 0.04).

\section{Cross-sectional analyses of maternal characteristics}

The distributions of the maternal characteristics in early and late pregnancy in our population are shown in (Table 2 and Table 3 ) respectively, stratified by the presence of depression or anxiety according to the HADS, using the predefined cut-off values. The results of the univariable and multivariable linear regression analyses, expressed as crude and adjusted $\beta$ coefficients with 95\% CIs, are shown in (Tables 4 and Table 5). In the univariable analyses, the majority of characteristics seemed to showed associations with the continuous HADS-D and/ or HADS-A scores, but many of these did not contribute independently to the multivariable models. Therefore, only the adjusted $\beta$ coefficients are described underneath. 
Citation: van de Loo KFE, Vlenterie R, Nikkels SJ, et al. (2017) Depression and Anxiety during Pregnancy: The Influence of Maternal Characteristics. J Mood Disord Ther 1(1):1-16

Table 2: Maternal characteristics of women with and without depression or anxiety in early pregnancy according to the scores on the HADS-depression (HADS-D) and HADS-anxiety (HADS-A) subscales.

\begin{tabular}{|c|c|c|c|c|}
\hline Maternal characteristics $^{1}$ & $\begin{array}{l}\text { HADS-D - } \\
N=2454(\%)\end{array}$ & $\begin{array}{l}\text { HADS-D + } \\
N=140(\%)\end{array}$ & $\begin{array}{l}\text { HADS-A - } \\
\mathrm{N}=2118(\%)\end{array}$ & $\begin{array}{l}\text { HADS-A + } \\
N=463(\%)\end{array}$ \\
\hline \multicolumn{5}{|l|}{ Demographics } \\
\hline \multicolumn{5}{|l|}{ Maternal age (years) } \\
\hline$\leq 30$ & $1291(52.6)$ & $71(50.7)$ & $1102(52.0)$ & $256(55.3)$ \\
\hline $30-34$ & $903(36.8)$ & $51(36.4)$ & $789(37.3)$ & $161(34.8)$ \\
\hline$\geq 35$ & $260(10.6)$ & $18(12.9)$ & $227(10.7)$ & $46(9.9)$ \\
\hline \multicolumn{5}{|l|}{ Level of education } \\
\hline High & $1829(74.5)$ & $91(65.0)$ & $1583(74.7)$ & $320(69.1)$ \\
\hline Intermediate & $561(22.9)$ & $40(28.6)$ & $487(23.0)$ & $119(25.7)$ \\
\hline Low & $54(2.2)$ & $9(6.4)$ & $38(1.8)$ & $24(5.2)$ \\
\hline \multicolumn{5}{|c|}{ Net household income (€/month) } \\
\hline$<2500$ & $322(13.1)$ & $29(20.7)$ & $282(13.3)$ & $69(14.9)$ \\
\hline$\geq 2500$ & $1741(70.9)$ & $81(57.9)$ & $1505(71.1)$ & $312(67.4)$ \\
\hline \multicolumn{5}{|l|}{ Population group } \\
\hline Dutch & $2374(96.7)$ & $132(94.3)$ & $2050(96.8)$ & $443(95.7)$ \\
\hline Non-Dutch & $73(3.0)$ & 81 & $60(2.8)$ & $20(4.3)$ \\
\hline \multicolumn{5}{|l|}{ Living together with partner } \\
\hline Yes & $1996(81.3)$ & $100(71.4)$ & $1740(82.2)$ & $350(75.6)$ \\
\hline No & $65(2.6)$ & $10(7.1)$ & $50(2.4)$ & $25(5.4)$ \\
\hline \multicolumn{5}{|l|}{ Medical history } \\
\hline \multicolumn{5}{|l|}{ Parity } \\
\hline Primiparity & $1410(57.5)$ & $67(47.9)$ & $1191(56.2)$ & $280(60.5)$ \\
\hline Multiparity & $1043(42.5)$ & $73(52.1)$ & $926(43.7)$ & $183(39.5)$ \\
\hline \multicolumn{5}{|l|}{ Pre-pregnancy BMI } \\
\hline Normal weight & $1670(68.1)$ & $89(63.6)$ & $1450(68.5)$ & $302(65.2)$ \\
\hline Underweight & $68(2.8)$ & $6(4.3)$ & $57(2.7)$ & $18(3.9)$ \\
\hline Overweight/obese & $539(22.0)$ & $35(25.0)$ & $467(22.0)$ & $102(22.0)$ \\
\hline \multicolumn{5}{|l|}{ Time to pregnancy ${ }^{2}$} \\
\hline$<1$ month & $649(28.6)$ & $34(32.1)$ & $574(29.3)$ & $115(27.8)$ \\
\hline $1-12$ months & $1324(58.3)$ & $59(55.7)$ & $1133(57.9)$ & $233(56.4)$ \\
\hline$>12$ months & $284(12.5)$ & $10(9.4)$ & $236(12.1)$ & $63(15.3)$ \\
\hline \multicolumn{5}{|l|}{ Previous miscarriages } \\
\hline Yes & $525(21.4)$ & $28(20.0)$ & $431(20.3)$ & $115(24.8)$ \\
\hline No & $1929(78.6)$ & $112(80.0)$ & $1687(79.7)$ & $348(75.2)$ \\
\hline \multicolumn{5}{|c|}{ Use of reproductive techniques } \\
\hline Natural conception & $2262(92.2)$ & $134(95.7)$ & $1969(93.0)$ & $417(90.1)$ \\
\hline Yes & $183(7.5)$ & $6(4.3)$ & $142(6.7)$ & $44(9.5)$ \\
\hline \multicolumn{5}{|l|}{ Chronic diseases } \\
\hline Yes & $426(17.4)$ & $28(20.1)$ & $360(17.0)$ & $91(19.7)$ \\
\hline No & 2005 (81.7) & 109 (77.9) & $1739(82.1)$ & $365(78.8)$ \\
\hline \multicolumn{5}{|c|}{ Pregnancy-related characteristics } \\
\hline \multicolumn{5}{|c|}{ Unplanned pregnancy } \\
\hline Yes & $175(7.1)$ & $34(24.3)$ & $156(7.4)$ & $48(10.4)$ \\
\hline No & $2272(92.6)$ & $106(75.7)$ & $1957(92.4)$ & $413(89.2)$ \\
\hline \multicolumn{5}{|l|}{ Infection during pregnancy } \\
\hline Yes & $1110(45.2)$ & $67(47.9)$ & $948(44.8)$ & $223(48.2)$ \\
\hline No & $1195(48.7)$ & $65(46.4)$ & $1046(49.4)$ & $210(45.4)$ \\
\hline \multicolumn{5}{|l|}{ Severe nausea } \\
\hline Yes & $486(19.8)$ & $52(37.1)$ & $421(19.9)$ & $111(24.0)$ \\
\hline No & $1333(54.3)$ & $56(40.0)$ & $1145(54.1)$ & 237 (51.2) \\
\hline Extreme fatigue & & & & \\
\hline
\end{tabular}


Citation: van de Loo KFE, Vlenterie R, Nikkels SJ, et al. (2017) Depression and Anxiety during Pregnancy: The Influence of Maternal Characteristics. J Mood Disord Ther 1(1):1-16

\begin{tabular}{|c|c|c|c|c|}
\hline Yes & $888(36.2)$ & $77(55.0)$ & $755(35.6)$ & $205(44.3)$ \\
\hline No & $931(37.9)$ & $31(22.1)$ & $815(38.5)$ & $142(30.7)$ \\
\hline \multicolumn{5}{|c|}{ Health behaviour during pregnancy } \\
\hline \multicolumn{5}{|l|}{ Smoking } \\
\hline Yes & $136(5.5)$ & $17(12.1)$ & $111(5.2)$ & $44(9.5)$ \\
\hline No & $2316(94.4)$ & $123(87.9)$ & $2006(94.7)$ & $418(90.3)$ \\
\hline \multicolumn{5}{|l|}{ Alcohol } \\
\hline Yes & 366 (14.9) & $29(20.7)$ & $314(14.8)$ & $76(16.4)$ \\
\hline No & $2083(84.9)$ & $110(78.6)$ & $1801(85.0)$ & $384(82.9)$ \\
\hline \multicolumn{5}{|c|}{ Caffeine consumption } \\
\hline Normal $(\leq 1400 \mathrm{mg})$ & $1814(73.9)$ & $105(76.0)$ & $1570(74.1)$ & $343(74.1)$ \\
\hline High (> 1400 mg) & $610(24.9)$ & $33(23.6)$ & $521(24.6)$ & $116(25.1)$ \\
\hline \multicolumn{5}{|l|}{ Physical exercise } \\
\hline No & $167(6.8)$ & $27(19.3)$ & $144(6.8)$ & $49(10.6)$ \\
\hline$<150 \mathrm{~m} /$ week & $1038(42.3)$ & $54(38.6)$ & $884(41.7)$ & $196(42.3)$ \\
\hline$\geq 150 \mathrm{~min} /$ week & $1247(50.8)$ & $56(40.0)$ & $1086(51.3)$ & $217(46.9)$ \\
\hline \multicolumn{5}{|c|}{ Psychosocial characteristics } \\
\hline \multicolumn{5}{|c|}{ Negative live event score } \\
\hline 0 & $1894(77.2)$ & $95(67.9)$ & $1647(77.8)$ & $331(71.5)$ \\
\hline$\leq 50$ & $424(17.3)$ & $32(22.9)$ & $365(17.2)$ & $91(19.7)$ \\
\hline$>50$ & $136(5.5)$ & $13(9.3)$ & $106(5.0)$ & $41(8.9)$ \\
\hline \multicolumn{5}{|c|}{$\begin{array}{l}\text { Use of medication for depression or } \\
\text { anxiety disorders }\end{array}$} \\
\hline Yes & $20(0.8)$ & $6(4.3)$ & $15(0.7)$ & $11(2.4)$ \\
\hline No & $2268(92.4)$ & $120(85.7)$ & $1978(93.4)$ & $397(85.7)$ \\
\hline \multicolumn{5}{|c|}{ History of depression } \\
\hline Yes & $552(22.5)$ & $50(35.7)$ & $426(20.1)$ & $172(37.1)$ \\
\hline No & $1893(77.1)$ & $90(64.3)$ & $1685(79.6)$ & $289(62.4)$ \\
\hline \multicolumn{5}{|c|}{ Family history of depression } \\
\hline Yes & $610(24.9)$ & $50(35.7)$ & $485(22.9)$ & $167(36.1)$ \\
\hline No & $1843(75.1)$ & $90(64.3)$ & $1632(77.1)$ & $296(63.9)$ \\
\hline
\end{tabular}

${ }^{1}$ Numbers of women within the strata do not add up to the total number of women for all variables due to missing values; ${ }^{2}$ Women with planned pregnancies only.

Table 3: Maternal characteristics of women with and without depression or anxiety in late pregnancy according to the scores on the HADS-depression (HADS-D) and HADS-anxiety (HADS-A) subscales.

\begin{tabular}{|c|c|c|c|c|}
\hline Maternal characteristics ${ }^{1}$ & $\begin{array}{l}\text { HADS-D - } \\
N=1910(\%)\end{array}$ & $\begin{array}{l}\text { HADS-D + } \\
N=213(\%)\end{array}$ & $\begin{array}{l}\text { HADS-A - } \\
N=1781(\%)\end{array}$ & $\begin{array}{l}\text { HADS-A + } \\
N=295(\%)\end{array}$ \\
\hline \multicolumn{5}{|l|}{ Demographics } \\
\hline \multicolumn{5}{|l|}{ Maternal age (years) } \\
\hline$\leq 30$ & $995(52.1)$ & $103(48.4)$ & $936(52.6)$ & $150(50.8)$ \\
\hline $30-34$ & $722(37.8)$ & $80(37.6)$ & $665(37.3)$ & $112(38.0)$ \\
\hline$\geq 35$ & $193(10.1)$ & $30(14.1)$ & $180(10.1)$ & $33(11.2)$ \\
\hline \multicolumn{5}{|l|}{ Level of education } \\
\hline High & $1486(77.8)$ & $126(59.2)$ & $1372(77.0)$ & $204(69.2)$ \\
\hline Intermediate & 378 (19.9) & $77(36.2)$ & $373(20.9)$ & $73(24.7)$ \\
\hline Low & $34(1.8)$ & $6(2.8)$ & $25(1.4)$ & $13(4.4)$ \\
\hline
\end{tabular}


Citation: van de Loo KFE, Vlenterie R, Nikkels SJ, et al. (2017) Depression and Anxiety during Pregnancy: The Influence of Maternal Characteristics. J Mood Disord Ther 1(1):1-16

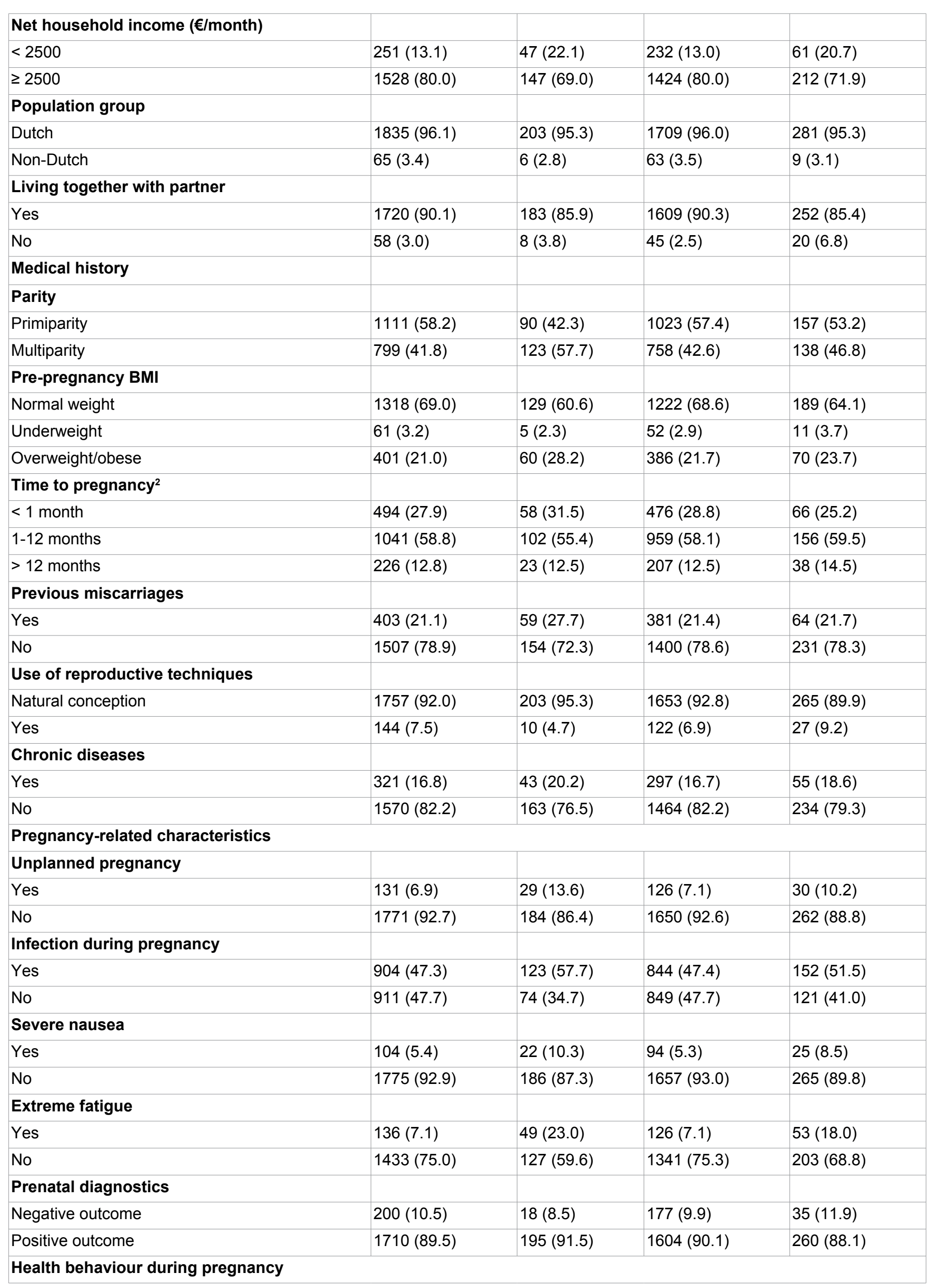


Citation: van de Loo KFE, Vlenterie R, Nikkels SJ, et al. (2017) Depression and Anxiety during Pregnancy: The Influence of Maternal Characteristics. J Mood Disord Ther 1(1):1-16

\begin{tabular}{|c|c|c|c|c|}
\hline Smoking & & & & \\
\hline Yes & $40(2.1)$ & $15(7.0)$ & $43(2.4)$ & $9(3.1)$ \\
\hline No & $1852(97.0)$ & $192(90.1)$ & $1719(96.5)$ & $280(94.9)$ \\
\hline \multicolumn{5}{|l|}{ Alcohol } \\
\hline Yes & $49(2.6)$ & $8(3.8)$ & $42(2.4)$ & $13(4.4)$ \\
\hline No & $1845(96.6)$ & $199(93.4)$ & $1721(96.6)$ & $277(93.9)$ \\
\hline \multicolumn{5}{|c|}{ Caffeine consumption } \\
\hline Normal $(\leq 1400 \mathrm{mg})$ & $1355(70.9)$ & $51(23.9)$ & $451(25.3)$ & $204(69.2)$ \\
\hline High (> 1400 mg) & $488(25.5)$ & $148(69.5)$ & $1266(71.1)$ & $75(25.4)$ \\
\hline \multicolumn{5}{|l|}{ Physical exercise } \\
\hline No & $154(8.1)$ & $38(17.8)$ & $155(8.7)$ & $36(12.2)$ \\
\hline$<150 \mathrm{~m} /$ week & $910(47.6)$ & $102(47.9)$ & $846(47.5)$ & $149(50.5)$ \\
\hline$\geq 150 \mathrm{~min} /$ week & $841(44.0)$ & $73(34.3)$ & 776 (43.6) & $110(37.3)$ \\
\hline \multicolumn{5}{|c|}{ Psychosocial characteristics } \\
\hline \multicolumn{5}{|c|}{ Negative live event score } \\
\hline 0 & $1346(70.5)$ & $131(61.5)$ & $1260(70.7)$ & $180(61.0)$ \\
\hline$\leq 50$ & $409(21.4)$ & $58(27.2)$ & $380(21.3)$ & $78(26.4)$ \\
\hline$>50$ & $155(8.1)$ & $24(11.3)$ & $141(7.9)$ & $37(12.5)$ \\
\hline \multicolumn{5}{|c|}{$\begin{array}{l}\text { Use of medication for depression or anxiety } \\
\text { disorders }\end{array}$} \\
\hline Yes & $10(0.5)$ & $1(0.5)$ & $5(0.3)$ & $5(1.7)$ \\
\hline No & $1864(97.6)$ & $201(94.4)$ & 1743 (97.9) & $276(93.6)$ \\
\hline \multicolumn{5}{|c|}{ History of depression } \\
\hline Yes & $411(21.5)$ & $74(34.7)$ & $348(19.5)$ & $122(41.4)$ \\
\hline No & $1491(78.1)$ & $136(63.8)$ & $1424(80.0)$ & $171(58.0)$ \\
\hline \multicolumn{5}{|c|}{ Family history of depression } \\
\hline Yes & 476 (24.9) & $64(30.0)$ & $435(24.4)$ & $93(31.5)$ \\
\hline No & $1431(74.9)$ & $147(69.0)$ & $1343(75.4)$ & $200(67.8)$ \\
\hline
\end{tabular}

${ }^{1}$ Numbers of women within the strata do not add up to the total number of women for all variables due to missing values; ${ }^{2}$ Women with planned pregnancies only.

Table 4: Cross-sectional models for the associations between maternal characteristics and continuous scores on the HADSdepression (HADS-D) and HADS-anxiety (HADS-A) subscales in early pregnancy.

\begin{tabular}{|c|c|c|c|c|}
\hline \multirow[t]{2}{*}{ Maternal characteristics } & \multicolumn{2}{|l|}{ HADS-D } & \multicolumn{2}{|l|}{ HADS-A } \\
\hline & Crude $\beta$ & Adjusted $\beta$ & Crude $\beta$ & Adjusted $\beta$ \\
\hline \multicolumn{5}{|l|}{ Demographics } \\
\hline \multicolumn{5}{|l|}{ Maternal age (years) } \\
\hline$\leq 30$ & Ref & - & Ref & Ref \\
\hline $30-34$ & $0.04(-0.17-0.24)$ & & $-0.14(-0.32-0.04)$ & $-0.07(-0.33-0.18)$ \\
\hline$\geq 35$ & $0.16(-0.16-0.49)$ & & $-0.01(-0.29-0.28)$ & $-0.50(-0.92--0.07)$ \\
\hline \multicolumn{5}{|l|}{ Level of education } \\
\hline High & Ref & Ref & Ref & - \\
\hline Intermediate & $0.53(0.30-0.76)$ & $0.27(-0.03-0.57)$ & $0.16(-0.04-0.36)$ & \\
\hline Low & $1.61(0.99-2.24)$ & $1.10(0.32-1.89)$ & $1.43(0.88-1.98)$ & \\
\hline $\begin{array}{l}\text { Net household income }<€ 2500 / \mathrm{mo}(€ / \\
\text { month) }\end{array}$ & $0.73(0.45-1.01)$ & - & $0.46(0.21-0.71)$ & - \\
\hline Non-Dutch & $0.74(0.19-1.29)$ & $1.11(0.38-1.83)$ & $0.39(-0.10-0.88)$ & - \\
\hline Not living together with partner & $1.25(0.68-1.82)$ & $1.45(0.71-2.18)$ & $1.24(0.75-1.74)$ & $1.12(0.35-1.89)$ \\
\hline \multicolumn{5}{|l|}{ Medical history } \\
\hline Multiparity & $0.56(-0.10-1.23)$ & - & $-0.07(-1.33--0.07)$ & - \\
\hline
\end{tabular}


Citation: van de Loo KFE, Vlenterie R, Nikkels SJ, et al. (2017) Depression and Anxiety during Pregnancy: The Influence of Maternal Characteristics. J Mood Disord Ther 1(1):1-16

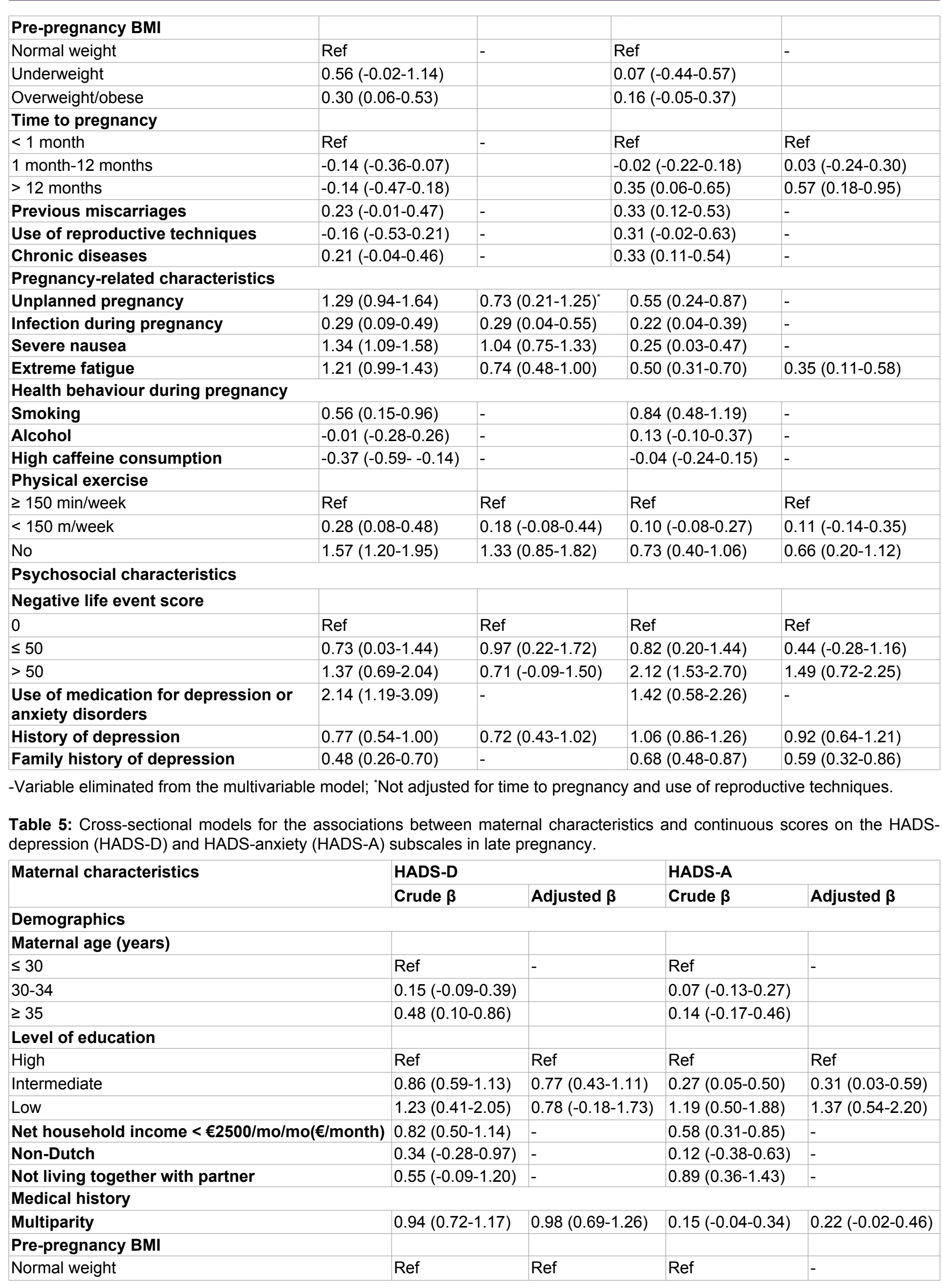


Citation: van de Loo KFE, Vlenterie R, Nikkels SJ, et al. (2017) Depression and Anxiety during Pregnancy: The Influence of Maternal Characteristics. J Mood Disord Ther 1(1):1-16

\begin{tabular}{|c|c|c|c|c|}
\hline Underweight & $0.35(-0.30-1.00)$ & $0.28(-0.50-1.06)$ & $0.24(-0.30-0.78)$ & \\
\hline Overweight/obese & $0.56(0.28-0.83)$ & $0.37(0.03-0.71)$ & $0.12(-0.11-0.34)$ & \\
\hline \multicolumn{5}{|l|}{ Time to pregnancy } \\
\hline$<1$ month & Ref & - & Ref & - \\
\hline 1 month-12 months & $-0.20(-0.47-0.06)$ & & $0.01(-0.22-0.22)$ & \\
\hline$>12$ months & $-0.17(-0.56-0.22)$ & & $0.08(-0.24-0.41)$ & \\
\hline Previous miscarriages & $0.38(0.10-0.65)$ & - & $0.04(-0.18-0.27)$ & - \\
\hline Use of reproductive techniques & $-0.16(-0.43-0.42)$ & - & $0.10(-0.27-0.46)$ & - \\
\hline Chronic diseases & $0.17(-0.13-0.46)$ & - & $0.07(-0.18-0.31)$ & - \\
\hline \multicolumn{5}{|l|}{ Pregnancy-related characteristics } \\
\hline Unplanned pregnancy & $1.09(0.66-1.51)$ & - & $0.30(-0.05-0.66)$ & - \\
\hline Infection during pregnancy & $0.41(0.18-0.64)$ & - & $0.33(0.14-0.52)$ & $0.22(-0.02-0.46)$ \\
\hline Severe nausea & $0.98(0.50-1.45)$ & - & $0.68(0.28-1.08)$ & - \\
\hline Extreme fatigue & $2.04(1.64-2.44)$ & $1.58(1.10-2.07)$ & $1.03(0.69-1.37)$ & $0.65(0.22-1.07)$ \\
\hline Negative prenatal diagnostics & $-0.06(-0.43-0.31)$ & - & $-0.06(-0.37-0.25)$ & - \\
\hline \multicolumn{5}{|l|}{ Health behaviour during pregnancy } \\
\hline Smoking & $1.43(0.72-2.14)$ & - & $0.53(-0.06-1.13)$ & - \\
\hline Alcohol & $0.10(-0.60-0.79)$ & - & $0.62(0.04-1.19)$ & - \\
\hline High caffeine consumption & $-0.25(-0.51-0.01)$ & - & $0.09(-0.13-0.30)$ & - \\
\hline \multicolumn{5}{|l|}{ Physical exercise } \\
\hline$\geq 150 \mathrm{~min} /$ week & Ref & Ref & Ref & Ref \\
\hline$<150 \mathrm{~m} /$ week & $0.32(0.09-0.56)$ & $0.21(-0.09-0.51)$ & $0.13(-0.07-0.33)$ & $0.27(0.02-0.52)$ \\
\hline No & $1.50(1.09-1.91)$ & $1.60(1.08-2.11)$ & $0.44(0.11-0.78)$ & $0.49(0.06-0.92)$ \\
\hline \multicolumn{5}{|l|}{ Psychosocial characteristics } \\
\hline \multicolumn{5}{|l|}{ Negative life event score } \\
\hline 0 & Ref & Ref & Ref & Ref \\
\hline$\leq 50$ & $0.33(-0.27-0.93)$ & $0.76(0.13-1.40)$ & $1.16(0.68-1.64)$ & $1.38(0.86-1.91)$ \\
\hline$>50$ & $0.76(0.06-1.47)$ & $0.72(-0.07-1.51)$ & $1.94(1.37-2.51)$ & $1.78(1.11-2.45)$ \\
\hline $\begin{array}{l}\text { Use of medication for depression or anxiety } \\
\text { disorders }\end{array}$ & $1.01(-0.55-2.57)$ & - & $1.98(0.65-3.31)$ & - \\
\hline History of depression & $0.87(0.60-1.13)$ & $0.60(0.26-0.93)$ & $1.16(0.94-1.38)$ & $1.08(0.80-1.37)$ \\
\hline Family history of depression & $0.44(0.18-0.70)$ & - & $0.39(0.17-0.60)$ & - \\
\hline
\end{tabular}

-Variable eliminated from the multivariable model.

\section{Demographics}

In early pregnancy only, women of 35 years of age or above had lower scores on the HADS-A subscale compared to women aged $<30$ years $(\beta-0.50,95 \%$ CI: $-0.92--0.07)$. Intermediate and low levels of education seemed to increase the HADS-D scores in both early and late pregnancy (low vs. high education: $\beta$ 1.10, 95\% CI: $0.32-1.89$ and $\beta 0.78$, 95\% CI: -0.18-1.73, respectively) and the HADS-A scores in late pregnancy only (low vs high education: $\beta 1.37,95 \%$ CI: 0.54-2.20). No associations were seen for net household income, but women from non-Dutch population groups reported higher scores on the HADS-D in early pregnancy compared to women from Dutch ancestry $(\beta 1.11,95 \%$ CI: 0.38-1.83). Women who did not live together with a partner had increased scores on both the HADS-D and the HADS-A in early pregnancy ( $\beta 1.45,95 \%$ CI: $0.71-2.18$ and $\beta 1.12,95 \%$ CI $0.35-1.89$ ).

\section{Medical history}

Multiparity was related to higher scores on the HADS-D and the HADS-A compared to nulliparity in late pregnancy
( $\beta$ 0.98, 95\% CI: 0.69-1.26 and $\beta$ 0.22, 95\% CI: $-0.02-0.46$ ). Women who were overweight or obese before pregnancy reported slightly higher scores on the HADS-D in late pregnancy compared to women with normal weight, whereas subfertile women (time to pregnancy $>12$ months) reported higher scores on the HADS-A in early pregnancy ( $\beta$ 0.57, 95\% CI: $0.18-0.95$ ), but not in late pregnancy. No associations were found for previous miscarriages, the use of reproductive techniques, and the presence of chronic diseases.

\section{Pregnancy-related characteristics}

Women with an unplanned pregnancy reported higher scores on the HADS-D in early, but not late pregnancy ( $\beta$ 0.73, 95\% CI: 0.21-1.25). Women who had an infection sometime during pregnancy reported slightly higher scores on the HADS-D in early pregnancy and on the HADS-A in late pregnancy than women without infections. Women with severe nausea had higher HADS-D scores in early pregnancy ( $\beta$ 1.04, 95\% CI: 0.75-1.33). Extreme fatigue seemed to affect the scores on the HADS-D and the HADS-A throughout pregnancy, with the largest 
Citation: van de Loo KFE, Vlenterie R, Nikkels SJ, et al. (2017) Depression and Anxiety during Pregnancy: The Influence of Maternal Characteristics. J Mood Disord Ther 1(1):1-16

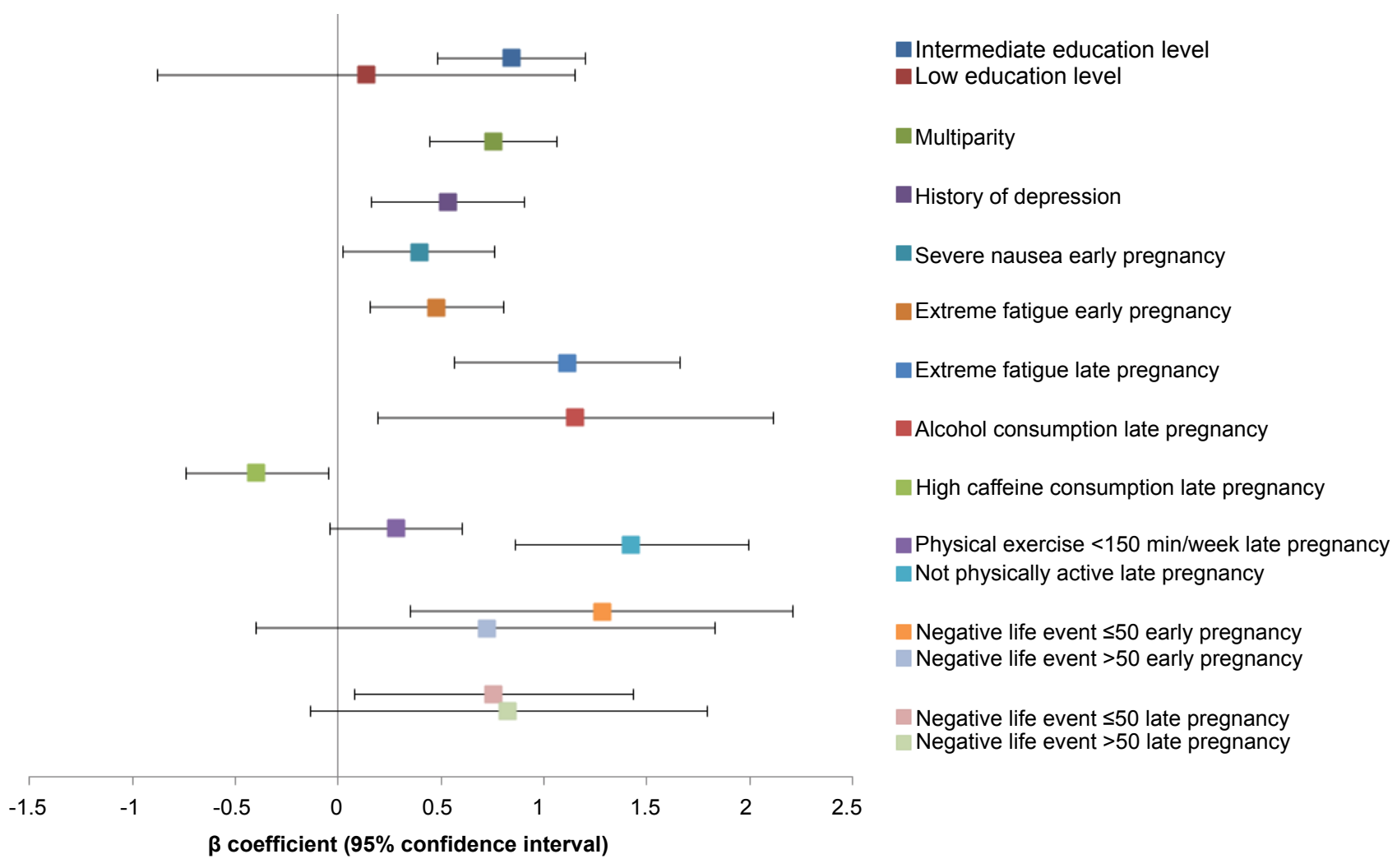

Figure 2: Longitudinal model for the association between maternal characteristics measured in early and late pregnancy and the continuous scores on the HADS-depression (HADS-D) subscale in late pregnancy.

score differences in late pregnancy $(\beta 1.58,95 \% \mathrm{CI}: 1.10-$ 2.07 and $\beta 0.65,95 \%$ CI: $0.22-1.07$ ). No independent association was found for a negative outcome of prenatal diagnostics.

\section{Health behavior during pregnancy}

No influences on the HADS scores were found for smoking, alcohol consumption, and high caffeine consumption in our population. However, not doing any physical exercise compared to exercising 150 minutes or more per week was associated with higher scores on both HADS subscales in early and late pregnancy, but especially with the HADS-D ( $\beta 1.33$, 95\% CI: $0.85-1.82$ and $\beta$ 1.60, 95\% CI: 1.08-2.11).

\section{Psychosocial characteristics}

Women who experienced negative life events reported higher scores on the HADS-D and HADS-A throughout pregnancy compared to women who did not experience any negative life event. The scores on the HADS-D were around 1.4 and 0.7 points higher for any negative life event in early and late pregnancy, respectively (negative life event score $>0$ : $\beta 1.35$, 95\% CI: $0.68-2.01$ and $\beta$ 0.74, 95\% CI: 0.04 $1.45)$, while the scores on the HADS-A were on average 1.5 and 1.8 points higher for major negative life events (score $>50)$ in early and late pregnancy, respectively $(\beta 1.49,95 \%$ CI: $0.72-2.25$ and $\beta 1.78,95 \%$ CI: 1.11-2.45). Although med- ication use for depression or anxiety disorders seemed to be associated with the HADS scores in the univariable analyses, no independent associations were found after adjustment for the other characteristics. However, women with a history of depression reported higher scores on both the HADS-D and the HADS-A in early ( $\beta 0.72,95 \%$ CI: $0.43-$ 1.02 and $\beta$ 0.92, 95\% CI: 0.64-1.21) and late pregnancy ( $\beta$ 0.60, 95\% CI: $0.26-0.93$ and $\beta$ 1.08, 95\% CI: $0.80-1.37$ ). A family history of depression only influenced the scores on the HADS-A in early pregnancy ( $\beta 0.59,95 \% \mathrm{CI}$ : 0.32-0.86).

\section{Longitudinal analyses}

When the characteristics of early and late pregnancy were combined in a longitudinal multivariable model, some characteristics measured in early pregnancy seemed to influenced the HADS scores in late pregnancy. As illustrated in (Figure 2), the following fixed and early pregnancy characteristics were risk factors for symptoms of depression in late pregnancy: intermediate versus high level of education (adjusted $\beta$ 0.84, 95\% CI: 0.48-1.20) but not low level of education, multiparity (adjusted $\beta 0.75,95 \%$ CI: 0.44-1.06), history of depression (adjusted $\beta$ 0.53, 95\% CI: 0.16-0.90), severe nausea (adjusted $\beta 0.39,95 \%$ CI: 0.02-0.76), extreme fatigue (adjusted $\beta$ 0.47, 95\% CI: 0.15-0.80), and negative life events (adjusted $\beta$ 1.28, 95\% CI: $0.35-2.21$ for score $\leq$ 50 and $\beta 0.72,95 \%$ CI: $-0.40-1.83$ for score $>50$ ). In addition, the already observed associations with extreme fatigue, 
Citation: van de Loo KFE, Vlenterie R, Nikkels SJ, et al. (2017) Depression and Anxiety during Pregnancy: The Influence of Maternal Characteristics. J Mood Disord Ther 1(1):1-16

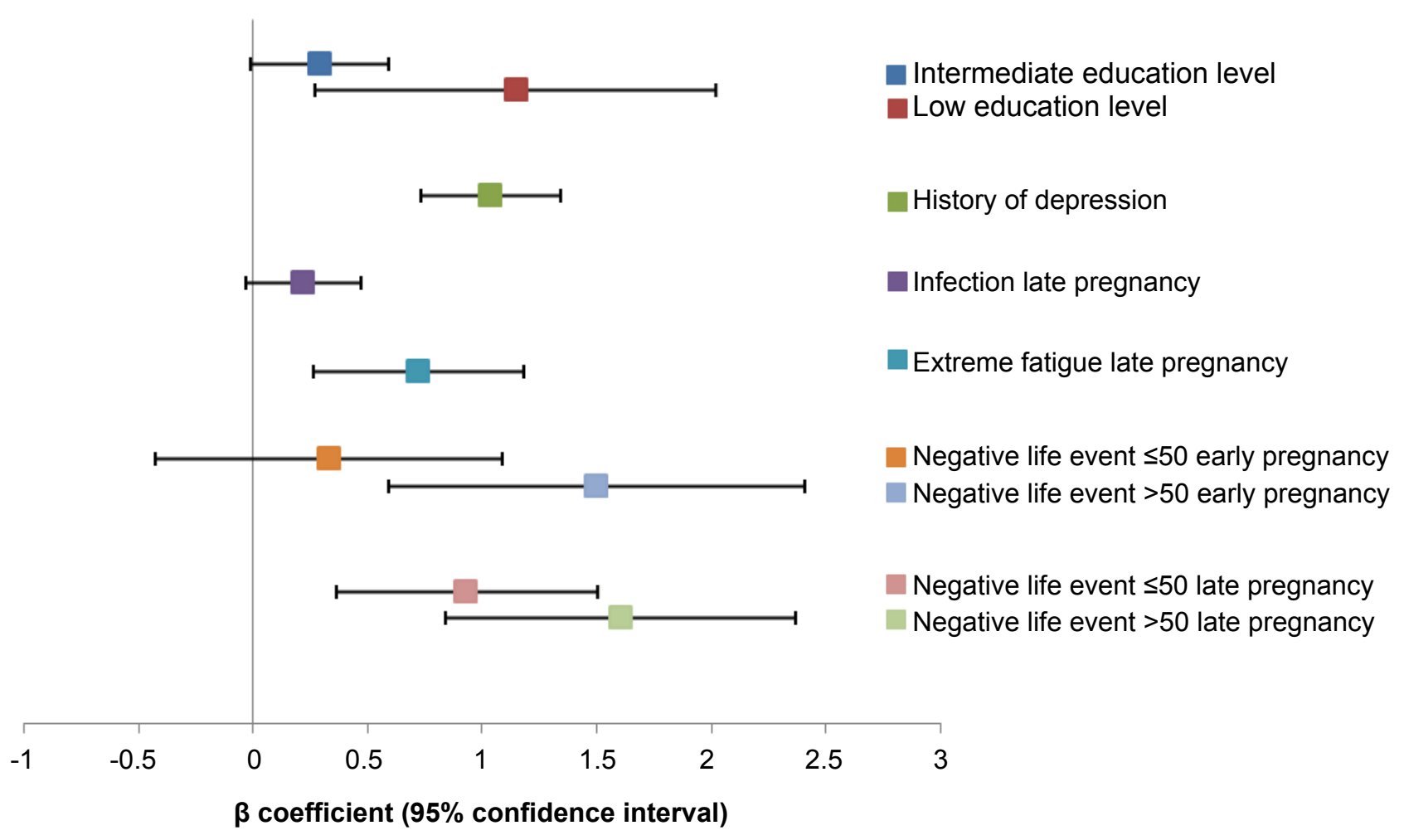

Figure 3: Longitudinal model for the associations between maternal characteristics measured in early and late pregnancy and the continuous scores on the HADS-anxiety (HADS-A) subscale in late pregnancy.

lack of exercise, and negative life events in late pregnancy remained in the longitudinal model, that also showed an adverse effect of alcohol consumption in late pregnancy ( $\beta$ 1.15, 95\% CI: $0.19-2.11)$ and a positive role for high caffeine consumption ( $\beta-0.40,95 \%$ CI: -0.74-0.05).

Risk factors for experiencing more symptoms of anxiety in late pregnancy were low or intermediate levels of education (adjusted $\beta$ 1.15, 95\% CI: 0.27-2.02 and $\beta$ $0.29,95 \%$ CI -0.01-0.59), history of depression (adjusted $\beta$ 1.04, 95\% CI: 0.73-1.34), and major negative life events in early pregnancy (adjusted $\beta$ 1.50, 95\% CI: 0.59-2.41), in addition to the already observed effects of infection, extreme fatigue, and negative life events in late pregnancy. No associations with a lack of exercise were found in the longitudinal model for the HADS-A. These results can be found in (Figure 3).

\section{Discussion}

This exploratory study revealed an increase in depression and a decrease in anxiety over the course of pregnancy assessed by the HADS in a large group of 2167 pregnant women from the general population in the Netherlands. The main maternal characteristics associated with depressive symptoms in early and/or late pregnancy were level of education, multiparity, having a history of depression, severe nausea, extreme fatigue, lack of physical exercise, negative life events, and alcohol consumption in late preg- nancy. Regarding anxiety, associations were mainly found with level of education, having a history of depression, extreme fatigue, lack of exercise, and having experienced negative life events. Being non-Dutch, not living with a partner, having an unplanned pregnancy, and having a long time to pregnancy influenced the depression and/or anxiety scores in early pregnancy only. This study investigated specific information regarding longitudinal changes in depression and anxiety during pregnancy, in combination with a large range of maternal factors that may be associated with symptoms of depression and anxiety.

A strong feature of this study is that the HADS was developed for physically ill patients and therefore filters out somatic symptoms of depression such as fatigue, insomnia, headaches, anergia, and loss of appetite $[42,43]$. This makes the HADS suitable for use during pregnancy regardless of the physical changes [31]. The HADS is a self-completed screening tool for depression and anxiety, however, and not a diagnostic instrument. By investigating the number of symptoms experienced, slight changes in mood can be detected, which provide more detailed results compared to solely investigating clinical diagnoses of depression and anxiety. Other strengths of this study are its prospective design, the large sample size, availability of information on many maternal characteristics throughout pregnancy, and measurements of symptoms of depression and anxiety at multiple time points during pregnancy using the same 
Citation: van de Loo KFE, Vlenterie R, Nikkels SJ, et al. (2017) Depression and Anxiety during Pregnancy: The Influence of Maternal Characteristics. J Mood Disord Ther 1(1):1-16

questionnaires. A limitation may be that the study sample consisted of relatively highly educated women, mainly from the Dutch population, which may hamper the generalizability of the results. However, this does not necessarily invalidate the associations found between the HADS scores and maternal characteristics $[44,45]$. Another limitation may be the self-reported nature of the data, but several validation studies comparing our data to a variety of medical records showed that the validity of most questionnaire items is good to excellent [46-48]. The drop-outs from our cohort had higher prevalence rates of depression and anxiety in early pregnancy compared to women who completed both questionnaires. This is a common problem and combined with the relatively high non-response rate among low educated women suggests that the prevalence rates of symptoms of depression and anxiety in Dutch pregnant women are most likely even higher than measured in this study $[36,49,50]$.

According to the HADS, the prevalence of depression in our population was $5.4 \%$ in early and $10.0 \%$ in late pregnancy, while the prevalence of anxiety was $17.9 \%$ and $14.2 \%$ in early and late pregnancy, respectively. Compared to other studies using the same questionnaire, the prevalence rates were lower compared to a Polish population (depression: $15.3 \%, 12.7 \%$, and $14.0 \%$ and anxiety: $27.4 \%, 23.9 \%$, and $29.9 \%$ in early, middle, and late pregnancy, respectively) [37], but higher compared to a Norwegian population (depression: $2.8 \%, 0.9 \%$, and $4.9 \%$, anxiety: $11.6 \%, 7.5 \%$, and $12.3 \%$ ) [51]. Another Dutch population study reported prevalence rates of depression and anxiety of $8.0 \%$ and $9.3 \%$ in mid-pregnancy, but these were assessed with the Brief Symptom Inventory (BSI) [52].

Previous results regarding the course of depression and anxiety throughout pregnancy were variable [31,33,34,36$38,53]$, and different types of depression and anxiety disorders may have different patterns pre- and perinatally [54]. In addition, the prevalence rates of depression are not likely to be influenced by differences between countries or regions, but rather by sociodemographic variables [34]. When investigating depression and anxiety, however, it is important to make a distinction between perceived symptoms and clinical diagnoses. A study by Halbreich suggested that the prevalence of mental disorders is not higher during pregnancy, only the prevalence of perceived symptoms [55]. Therefore, the increase in depressive symptoms over time found in our study may partly be explained by higher demands in late pregnancy compared to early pregnancy [34]. Regarding anxiety, we observed a decrease in general symptoms of anxiety over pregnancy. This may be due to different types of anxiety having different patterns over time. Pregnancy-specific anxiety, for example, is seen as a distinct anxiety response in pregnant women [30,56], but other types of anxiety may play a role as well. For future research, we suggest to focus on the presentation of depression and anxiety, as well as on the biological basis for depression and anxiety in both pregnant and non-pregnant women.

Several maternal characteristics were associated with depression or anxiety in early and/or late pregnancy, and with the increase or decrease in depression and anxiety, respectively. As they were partly associated with different factors, however, depression and anxiety may have a different pathogenesis [31]. In our study, multiparity was a risk factor for depression and anxiety, while results from previous studies are inconsistent [57]. One study reported no effect of parity on depression or anxiety, but found that multiparous women reported higher interpersonal risk factors for anxiety and depression i.e. lower levels of social support and marital satisfaction, with less maladaptive attitudes toward motherhood [58]. Therefore, possible moderating factors should be included in further investigations of the influence of multiparity on depression and anxiety. Severe nausea in early pregnancy was also associated with depression only, whereas previous literature showed that severe nausea with vomiting (hyperemesis gravidarum) was related to both depression and anxiety during pregnancy [59-64].

In line with previous research [65], extreme fatigue was associated with depression, but in our study with anxiety as well. Fatigue is a common symptom of pregnancy and seems less likely to be a sign of depression in pregnant women compared to non-pregnant women [66]. Therefore, extreme fatigue during pregnancy may be considered as a risk factor for depression and anxiety, rather than as a symptom of depression. In the general population, physical exercise may have a positive effect on symptoms of depression and anxiety [67]. Less is known about this topic during pregnancy, although being physically active has been related to fewer symptoms of depression pre- and postnatally [68-70], and regular physical activity was reported to improve anxiety and depression during pregnancy [71]. In our cross-sectional analyses, a lack of physical activity was associated with symptoms of depression and anxiety in both early and late pregnancy, and with depression only in the longitudinal model. It is as yet unclear whether these associations point towards low physical activity as a cause or a consequence of depression and/or fatigue during pregnancy. Having a history of depression was a risk factor for both depression and anxiety in our study, which is in line with previous studies $[32,57,58]$. Negative life events, which were rated into a robust measure of perceived stress, were also associated with higher prevalence rates of depression and anxiety. Life events are likely to be a source of stress and seem to be associated with depression and anxiety during pregnancy [57,72]. However, the impact of life events on depression and anxiety is not straightforward. First of all, social support and coping strategies play-among others-a 
Citation: van de Loo KFE, Vlenterie R, Nikkels SJ, et al. (2017) Depression and Anxiety during Pregnancy: The Influence of Maternal Characteristics. J Mood Disord Ther 1(1):1-16

moderating role on the impact of stress [6,70]. Secondly, the impact of chronic (repeated stressful minor events and daily hassles) versus acute stress should be differentiated, as chronic stress seems to have a more detrimental impact on fetal development compared to acute stress [6]. Finally, the timing of exposure to the stressor is important. Some studies reported that events occurring before 32 weeks were perceived as more stressful than events occurring later in pregnancy [73]. The first half of pregnancy may be a more vulnerable period, as critical life events were linked to increased cortisol levels in early pregnancy only [74]. However, the impact of a major life event on pregnancy outcome (e.g. shortened gestational age) may be greatest during the $5^{\text {th }}$ or $6^{\text {th }}$ month of pregnancy [75]. To determine the influence of life events on depression and anxiety, more research is needed in the above mentioned areas.

In conclusion, the prevalence rates of depression were higher in late pregnancy compared to early pregnancy in our study population. The prevalence rates of anxiety were relatively high, but decreased somewhat from early to late pregnancy. This study identified several risk factors for symptoms of depression and/or anxiety in early and late pregnancy, as well as some factors in early pregnancy that were still associated with depression and anxiety in late pregnancy. Screening for these risk factors in early pregnancy is important to provide adequate support to women at risk at an early stage. As a result, proper counseling and therapy during pregnancy may lead to prevention of adverse maternal and child health outcomes.

\section{Acknowledgements}

We want to thank all women who participated in the PRIDE Study and all prenatal health care providers who helped us with the inclusion of pregnant women in this ongoing cohort study. This study was partly funded by the Lung Foundation Netherlands (project 3.4.10.007) and by the Netherlands Organization for Health Research and Development, Program Rational Pharmacotherapy.

\section{References}

1. Alder J, Fink N, Bitzer J, et al. (2007) Depression and anxiety during pregnancy: a risk factor for obstetric, fetal and neonatal outcome? A critical review of the literature. J Matern Fetal Neonatal Med 20: 189-209.

2. Barker ED, Jaffee SR, Uher R, et al. (2011) The contribution of prenatal and postnatal maternal anxiety and depression to child maladjustment. Depress Anxiety 28: 696-702.

3. Betts KS, Williams GM, Najman JM, et al. (2014) Maternal depressive, anxious, and stress symptoms during pregnancy predict internalizing problems in adolescence. Depress Anxiety 31: 9-18.

4. Dunkel Schetter C, Tanner L (2012) Anxiety, depression and stress in pregnancy: implications for mothers, children, research, and practice. Curr Opin Psychiatry 25: 141-148.
5. Glover V (2014) Maternal depression, anxiety and stress during pregnancy and child outcome; what needs to be done. Best Pract Res Clin Obstet Gynaecol 28: 25-35.

6. Graignic-Philippe R, Dayan J, Chokron S, et al. (2014) Effects of prenatal stress on fetal and child development: a critical literature review. Neurosci Biobehav Rev 43: 137-162.

7. Grigoriadis S, VonderPorten EH, Mamisashvili L, et al. (2013) The impact of maternal depression during pregnancy on perinatal outcomes: a systematic review and meta-analysis. J Clin Psychiatry 74: e321-e341.

8. Kim DR, Bale TL, Epperson CN, et al. (2015) Prenatal programming of mental illness: current understanding of relationship and mechanisms. Curr Psychiatry Rep 17: 5.

9. Chang HY, Keyes KM, Lee KS, et al. (2014) Prenatal maternal depression is associated with low birth weight through shorter gestational age in term infants in Korea. Early Hum Dev 90: 15-20.

10. Grote NK, Bridge JA, Gavin AR, et al. (2010) A meta-analysis of depression during pregnancy and the risk of preterm birth, low birth weight, and intrauterine growth restriction. Arch Gen Psychiatry 67: 1012-1024.

11. Ibanez G, Charles MA, Forhan A, et al. (2012) Depression and anxiety in women during pregnancy and neonatal outcome: data from the EDEN mother-child cohort. Early Hum Dev 88: 643-649.

12. Liu C, Cnattingius S, Bergstrom M, et al. (2016) Prenatal parental depression and preterm birth: a national cohort study. BJOG 123: 1973-1982.

13. Phillips DI, Jones A, Goulden PA, et al. (2006) Birth weight, stress, and the metabolic syndrome in adult life. Ann N Y Acad Sci 1083: 28-36.

14. Saigal S, Doyle LW (2008) An overview of mortality and sequelae of preterm birth from infancy to adulthood. Lancet 371: 261-269.

15. Kingston D, Tough S, Whitfiel $\mathrm{H}$, et al. (2012) Prenatal and postpartum maternal psychological distress and infant development: a systematic review. Child Psychiatry Hum Dev 43: 683-714.

16. Weinstock T, Shoham-Vardi I, Glasser S, et al. (2015) Fetal sex modifies effects of prenatal stress exposure and adverse birth outcomes. Stress 18: 49-56.

17. Zijlmans MA, Riksen-Walraven JM, de Weerth C, et al. (2015) Associations between maternal prenatal cortisol concentrations and child outcomes: A systematic review. Neurosci Biobehav Rev 53: 1-24.

18. Toohey J (2012) Depression during pregnancy and postpartum. Clin Obstet Gynecol 55: 788-797.

19. Alipour Z, Lamyian M, Hajizadeh E, et al. (2012) Anxiety and fear of childbirth as predictors of postnatal depression in nulliparous women. Women Birth 25: e37-e43.

20. Edwards B, Galletly C, Semmler-Booth T, et al. (2008) Does antenatal screening for psychosocial risk factors predict postnatal depression? A follow-up study of 154 women in Adelaide, South Australia. Aust N Z J Psychiatry 42: 5155.

21. Lee DT, Chung TK (2007) Postnatal depression: an update. Best Pract Res Clin Obstet Gynaecol 21: 183-191. 
Citation: van de Loo KFE, Vlenterie R, Nikkels SJ, et al. (2017) Depression and Anxiety during Pregnancy: The Influence of Maternal Characteristics. J Mood Disord Ther 1(1):1-16

22. Leigh B, Milgrom J (2008) Risk factors for antenatal depression, postnatal depression and parenting stress. BMC Psychiatry 8: 24.

23. Milgrom J, Gemmill AW, Bilszta JL, et al. (2008) Antenatal risk factors for postnatal depression: a large prospective study. J Affect Disord 108: 147-157.

24. Ngai FW, Ngu SF (2015) Predictors of maternal and paternal depressive symptoms at postpartum. J Psychosom Res 78: 156-161.

25. Serati M, Redaelli M, Buoli M, et al. (2016) Perinatal Major Depression Biomarkers: A systematic review. J Affect Disord 193: 391-404.

26. Sutter-Dallay AL, Giaconne-Marcesche V, Glatigny-Dallay E, et al. (2004) Women with anxiety disorders during pregnancy are at increased risk of intense postnatal depressive symptoms: a prospective survey of the MATQUID cohort. Eur Psychiatry 19: 459-463.

27. Yim IS, Glynn LM, Dunkel-Schetter C, et al. (2009) Risk of postpartum depressive symptoms with elevated corticotropin releasing hormone in human pregnancy. Arch Gen Psychiatry 66: 162-169.

28. Najman JM, Plotnikova M, Williams GM, et al. (2016) Trajectories of maternal depression: a 27-year population-based prospective study. Epidemiol Psychiatr Sci 26: 79-88.

29. Bayrampour H, McDonald S, Tough S, et al. (2015) Risk factors of transient and persistent anxiety during pregnancy. Midwifery 31: 582-589.

30. Brunton RJ, Dryer R, Saliba A, et al. (2015) Pregnancy anxiety: A systematic review of current scales. J Affect Disord 176: $24-34$.

31. Lee AM, Lam SK, Sze Mun Lau SM, et al. (2007) Prevalence, course, and risk factors for antenatal anxiety and depression. Obstet Gynecol 110: 1102-1112.

32. Nasreen HE, Kabir ZN, Forsell Y, et al. (2011) Prevalence and associated factors of depressive and anxiety symptoms during pregnancy: a population based study in rural Bangladesh. BMC Womens Health 11: 22.

33. Gavin NI, Gaynes BN, Lohr KN, et al. (2005) Perinatal depression: a systematic review of prevalence and incidence. Obstet Gynecol 106: 1071-1083.

34. Bennett HA, Einarson A, Taddio A, et al. (2004) Prevalence of depression during pregnancy: systematic review. Obstet Gynecol 103: 698-709.

35. Verbeek T, Arjadi R, Vendrik JJ, et al. (2015) Anxiety and depression during pregnancy in Central America: a cross-sectional study among pregnant women in the developing country Nicaragua. BMC Psychiatry 15: 292.

36. van Bussel JC, Spitz B, Demyttenaere K, et al. (2009) Anxiety in pregnant and postpartum women. An exploratory study of the role of maternal orientations. J Affect Disord 114: 232-242.

37. Morylowska-Topolska J, Makara-Studzinska M, Kotarski J, et al. (2014) The influence of sociodemografic and medical variables on severity of anxiety and depressive symptoms during particular trimesters of pregnancy. Psychiatr Pol 48: 173-186.

38. Glynn LM, Schetter CD, Hobel CJ, et al. (2008) Pattern of perceived stress and anxiety in pregnancy predicts preterm birth. Health Psychol 27: 43-51.
39. van Gelder MM, Bretveld RW, Roukema J, et al. (2013) Rationale and design of the PRegnancy and Infant DEvelopment (PRIDE) Study. Paediatr Perinat Epidemiol 27: 34-43.

40. Zigmond AS, Snaith RP (1983) The hospital anxiety and depression scale. Acta Psychiatr Scand 67: 361-370.

41. Spinhoven P, Ormel J, Sloekers PP, et al. (1997) A validation study of the Hospital Anxiety and Depression Scale (HADS) in different groups of Dutch subjects. Psychol Med 27: 363-370.

42. Bjelland I, Dahl AA, Haug TT, et al. (2002) The validity of the Hospital Anxiety and Depression Scale. An updated literature review. J Psychosom Res 52: 69-77.

43. Herrmann C (1997) International experiences with the Hospital Anxiety and Depression Scale--a review of validation data and clinical results. J Psychosom Res 42: 17-41.

44. Nohr EA, Frydenberg M, Henriksen TB, et al. (2006) Does low participation in cohort studies induce bias?. Epidemiology 17: 413-418.

45. Nilsen RM, Vollset SE, Gjessing HK, et al. (2009) Self-selection and bias in a large prospective pregnancy cohort in Norway. Paediatr Perinat Epidemiol 23: 597-608.

46. van Gelder MM, Schouten NP, Merkus PJ, et al. (2015) Using Web-Based Questionnaires and Obstetric Records to Assess General Health Characteristics Among Pregnant Women: A Validation Study. J Med Internet Res 17: e149.

47. Van Gelder MM, Vorstenbosch S, te Winkel B, et al. (2016) Using web-based questionnaires to assess medication use during pregnancy: a validation study in two prospective cohort studies. Pharmacoepidemiol Drug Saf 25: 3-681.

48. Van Gelder MM, Vorstenbosch S, te Winkel B, et al. (2016) Validity of a web-based questionnaire to assess perinatal outcome. Pharmacoepidemiol Drug Saf 2: 3-681.

49. Austin MP, Tully L, Parker G, et al. (2007) Examining the relationship between antenatal anxiety and postnatal depression. J Affect Disord 101: 169-174.

50. Britton JR (2008) Maternal anxiety: course and antecedents during the early postpartum period. Depress Anxiety 25: $793-800$.

51. Berle JO, Mykletun A, Daltveit AK, et al. (2005) Neonatal outcomes in offspring of women with anxiety and depression during pregnancy. A linkage study from The Nord-Trondelag Health Study (HUNT) and Medical Birth Registry of Norway. Arch Womens Ment Health 8: 181-189.

52. Guxens M, Sonnenschein-van der Voort AM, Tiemeier $H$, et al. (2014) Parental psychological distress during pregnancy and wheezing in preschool children: the Generation R Study. J Allergy Clin Immunol 133: 59-67.

53. Kisilevsky BS, Reissland N (2016) Fetal Development: Research on Brain and Behaviour, Environmental Influences and Emerging Technologies. 229-265.

54. Martini J, Petzoldt J, Einsle F, et al. (2015) Risk factors and course patterns of anxiety and depressive disorders during pregnancy and after delivery: a prospective-longitudinal study. J Affect Disord 175: 385-395.

55. Halbreich U (2004) Prevalence of mood symptoms and depressions during pregnancy: implications for clinical practice and research. CNS Spectr 9: 177-184. 
Citation: van de Loo KFE, Vlenterie R, Nikkels SJ, et al. (2017) Depression and Anxiety during Pregnancy: The Influence of Maternal Characteristics. J Mood Disord Ther 1(1):1-16

56. Huizink AC, Mulder EJ, Robles de Medina PG, et al. (2004) Is pregnancy anxiety a distinctive syndrome?. Early Hum Dev 79: 81-91.

57. Biaggi A, Conroy S, Pawlby S, et al. (2016) Identifying the women at risk of antenatal anxiety and depression: A systematic review. J Affect Disord 191: 62-77.

58. Sockol LE, Battle CL (2015) Maternal attitudes, depression, and anxiety in pregnant and postpartum multiparous women. Arch Womens Ment Health 18: 585-593.

59. Aksoy H, Aksoy U, Karadag OI, et al. (2015) Depression levels in patients with hyperemesis gravidarum: a prospective case-control study. Springerplus 4: 34.

60. Annagur BB, Kerimoglu OS, Gunduz S, et al. (2014) Are there any differences in psychiatric symptoms and eating attitudes between pregnant women with hyperemesis gravidarum and healthy pregnant women?. J Obstet Gynaecol Res 40: 1009-1014.

61. Annagur BB, Tazegul A, Gunduz S, et al. (2013) Do psychiatric disorders continue during pregnancy in women with hyperemesis gravidarum: a prospective study. Gen Hosp Psychiatry 35: 492-496.

62. Hizli D, Kamalak Z, Kosus A, et al. (2012) Hyperemesis gravidarum and depression in pregnancy: is there an association? J Psychosom Obstet Gynaecol 33: 171-175.

63. Simsek Y, Celik O, Yilmaz E, et al. (2012) Assessment of anxiety and depression levels of pregnant women with hyperemesis gravidarum in a case-control study. J Turk Ger Gynecol Assoc 13: 32-36.

64. Uguz F, Gezginc K, Kayhan F, et al. (2012) Is hyperemesis gravidarum associated with mood, anxiety and personality disorders: a case-control study. Gen Hosp Psychiatry 34: 398-402.

65. Cheng CY, Pickler RH (2014) Perinatal stress, fatigue, depressive symptoms, and immune modulation in late pregnancy and one month postpartum. Scientific World Journal 2014: 652630
66. Nylen KJ, Williamson JA, O'Hara MW, et al. (2013) Validity of somatic symptoms as indicators of depression in pregnancy. Arch Womens Ment Health 16: 203-210.

67. Pedersen BK, Saltin B (2015) Exercise as medicine - evidence for prescribing exercise as therapy in 26 different chronic diseases. Scand J Med Sci Sports 3: 1-72.

68. Henriksson-Larsen K (1999) Training and sports competition during pregnancy and after childbirth. Physical training is beneficial for mother and child. Lakartidningen 96: 20972100.

69. Nordhagen IH, Sundgot-Borgen J (2002) Physical activity among pregnant women in relation to pregnancy-related complaints and symptoms of depression. Tidsskr Nor Laegeforen 122: 470-474.

70. Robledo-Colonia AF, Sandoval-Restrepo N, Mosquera-Valderrama YF, et al. (2012) Aerobic exercise training during pregnancy reduces depressive symptoms in nulliparous women: a randomised trial. J Physiother 58: 9-15.

71. Shivakumar G, Brandon AR, Snell PG, et al. (2011) Antenatal depression: a rationale for studying exercise. Depress Anxiety 28: 234-242.

72. Lancaster CA, Gold KJ, Flynn HA, et al. (2010) Risk factors for depressive symptoms during pregnancy: a systematic review. Am J Obstet Gynecol 202: 5-14.

73. Glynn LM, Schetter CD, Wadhwa PD, et al. (2004) Pregnancy affects appraisal of negative life events. J Psychosom Res 56: 47-52.

74. Rothenberger SE, Moehler E, Reck C, et al. (2011) Prenatal stress: course and interrelation of emotional and physiological stress measures. Psychopathology 44: 60-67.

75. Class QA, Lichtenstein P, Langstrom N, et al. (2011) Timing of prenatal maternal exposure to severe life events and adverse pregnancy outcomes: a population study of 2.6 million pregnancies. Psychosom Med 73: 234-241. 